\title{
Common fixed point of generalized cyclic Banach algebra contractions and Banach algebra Kannan types of mappings on cone quasi metric spaces
}

\author{
Sahar Mohamed Ali Abou Bakr \\ Department of Mathematics, Faculty of Science, Ain Shams University, Cairo, Egypt.
}

\begin{abstract}
This paper proves the existence of a unique common fixed point of two self mappings defined on complete cone quasi metric space $\mathfrak{C}$ with respect to Banach algebra, consequently in particular, it proves the existence of only one fixed point of a generalized cyclic Banach algebra contraction and a cyclic Banach algebra Kannan type mappings with respect to a couple of non empty subsets $(A, B)$ of a complete cone quasi metric space $\mathfrak{C}$. These existences extend the fixed point results of the attached references and then generalized the corresponding classical results in usual Banach spaces as well.
\end{abstract}

Keywords: Quasi metric spaces, fixed point theorems, $\{a, b, c\}$ generalized contractions, generalized $\phi$ weak contractions, cyclic contraction mappings.

2010 MSC: 47H09, 47H10.

(C)2019 All rights reserved.

\section{Introduction and preliminaries}

In 2007, Huang and Zhang [16] introduced cone metric spaces as generalization of metric spaces, they carefully defined convergence and completeness in these spaces and proved some fixed point theorems in this setting. The distance $d(x, y)$ of two elements $x$ and $y$ in a cone metric space $(X, d)$ is defined to be a vector in a cone of an ordered Banach space $(E, \leqslant)$, and a mapping $T: X \rightarrow X$ is said to be contractive if there is a constant $k \in[0,1)$ such that

$$
d(T x, T y) \leqslant k d(x, y), \quad \forall x, y \in X .
$$

In 2010, Haghi et al. [13] showed that some generalizations in fixed point theory are really consequences of Huang and Zhang results.

It no longer becomes better generalization because in 2012, Çakally et al. [5] obtained that any cone metric space $(X, d)$ is equivalent to the usual metric space $\left(X, d^{\star}\right)$, where the real-valued metric function $\mathrm{d}^{\star}$ is defined by a nonlinear scalarization function, one also can see [1, 6].

In 2013, Liu and $\mathrm{Xu}$ [18] introduce the concept of cone metric spaces with Banach algebras, they replaced Banach spaces $E$ by Banach algebras $\mathcal{A}$ as the underlying spaces of cone metric spaces. With

Email address: saharm_ali@yahoo.com; saharm_ali@sci.asu.edu.eg (Sahar Mohamed Ali Abou Bakr)

doi: $10.22436 /$ jnsa.012.10.03

Received: 2019-02-04 Revised: 2019-04-03 Accepted: 2019-04-07 
this modification, they proved some fixed point theorems of generalized Lipschitz mappings with weaker conditions on generalized Lipschitz constants (the constant $k$ is a vector in a normal cone of the Banach algebra and the essential conditions on the contraction constant $k$ are neither order relations nor norm relations, but spectrum radius). A well formulated example shows that their main results concerning the fixed point theorems in the setting of cone metric spaces with Banach algebras are more useful than the standard results in cone metric spaces presented in the literature.

A Banach algebra is a set $\mathcal{A}$ admitting three operations, scalar multiplication, vector addition + , and vector multiplication o such that

1. $\mathcal{A}$ is a complex Banach space $(\mathcal{A},\|\|$.$) with respect to vector addition and vector multiplication;$

2. $\mathcal{A}$ is a ring with respect to vector addition and vector multiplication;

3. for a scalar $\mu \in \mathcal{C}$ and any two vectors $y$ and $z \in \mathcal{A}, \mu($ yoz $)=(\mu \mathrm{y}) \mathrm{o} z=$ yo $(\mu z)$;

4. for any two vectors $y$ and $z \in \mathcal{A}$, $\| y$ o $z\|\leqslant\| y\|\| z \|$.

In this paper, if $\mathcal{A}$ has identity element with respect to multiplication, then the identity element with respect to multiplication will be denoted by $\Delta$, while the neutral element with respect to addition will be denoted by $\Theta$.

The spectral radius of $y \in \mathcal{A}$ denoted by $\rho(y)$ is defined as:

$$
\rho(y):=\sup \{\lambda \in \mathbb{C}: y-\lambda \Delta \text { has no multiplicative inverse element }\} \text {. }
$$

The following is a well known lemma [20].

Lemma 1.1. If $\mathcal{A}$ is a Banach algebra with a unit $\Delta$ and $y \in \mathcal{A}$, then

1. we have

$$
\rho(y)=\lim _{n \rightarrow \infty} \sqrt[n]{\left\|y^{n}\right\|}=\inf _{n \in \mathbb{N}} \sqrt[n]{\left\|y^{n}\right\|}
$$

2. if $\rho(y)<1$, then $\Delta-\mathrm{y}$ has a multiplicative inverse $(\Delta-y)^{-1}$. Moreover $(\Delta-y)^{-1}=\sum_{n=0}^{\infty} y^{n}$, where the series is norm convergent.

Remark 1.2. If $x$ and $y$ are two commuting elements in a Banach algebra $\mathcal{A}$, then $\rho($ yo $x) \leqslant \rho(y) \rho(x)$. In fact; $\rho($ yox $)=\lim _{n \rightarrow \infty} \|[\text { yox }]^{n}\left\|^{\frac{1}{n}}=\lim _{n \rightarrow \infty}\right\| y^{n} o x^{n} \|^{\frac{1}{n}} \leqslant \lim _{n \rightarrow \infty}\left[\left\|y^{n}\right\|\left\|x^{n}\right\|\right]^{\frac{1}{n}}=\lim _{n \rightarrow \infty}\left[\left\|y^{n}\right\|^{\frac{1}{n}}\left\|x^{n}\right\|^{\frac{1}{n}}\right]=$ $\rho(y) \rho(x)$.

A subset $\mathrm{C}$ of $\mathcal{A}$ is a cone in $\mathcal{A}$ iff

1. $C$ is non-empty closed and $\{\Theta, \Delta\} \subset C$;

2. $\alpha C+\beta C \subset C$ for all non-negative real numbers $\alpha, \beta$;

3. $\mathrm{C}^{2}=\mathrm{C} \times \mathrm{C} \subset \mathrm{C}$;

4. $\mathrm{C} \cap-\mathrm{C}=\{\Theta\}$.

A cone $\mathrm{C}$ in a Banach algebra $\mathcal{A}$ induces an ordered relation

$$
y \leqslant z \Longleftrightarrow z-y \in C
$$

In [18] one also considers the order relations

$$
y \lesseqgtr z \Longleftrightarrow(z-y \in C \text { and } y \neq z), \quad \text { and } \quad y<z \Longleftrightarrow z-y \in \text { int } C,
$$

where int $\mathrm{C}$ is the set of all interior points of $\mathrm{C}$. Some authors denote $\leqslant$ by $\ll$.

A sequence $\left\{z_{n}\right\}_{n \in N}$ in $\mathcal{A}$ is bounded above by $z \in \mathcal{A}$ iff

$$
z_{\mathrm{n}} \ll z, \quad \forall \mathrm{n} \in \mathbb{N} .
$$


The cone $C$ is called normal if there is a number $M>0$ such that for all $x, y \in \mathcal{A}$,

$$
\Theta \leqslant x \leqslant y \Longrightarrow\|x\| \leqslant M\|y\|
$$

In 2016, Huang and Radenovic [15] generalized the concept of [18], they introduced a more general cone $b$ metric space over a Banach algebra and proved some fixed point results of generalized Lipschitz mappings concerned with this space.

In 2017, George et-al. [12] later generalized Huang and Radenovic's concept as they introduce rectangular cone b-metric space over a Banach algebra, and proved both Banach contraction principle and weak Kannan contraction principle in these spaces, they provided examples supporting the superiority of their results.

On the other side, in 2003, Kirk et-al. [17] introduced the concept of contractive types of mapping satisfying cyclical condition and proved also some related fixed point results.

In 2008, Choudhury [7] proved the existence of (fp) for the weak $\psi-\phi$ contraction mappings.

In 2012, Aydi et al. [4] showed that some of these fixed points theorems are particular of previous existing theorems.

In 2013, Haghi et al. [14] took care of Cauchy-ness, convergent, and completeness definitions in partial metric spaces, and they showed that fixed point generalizations to partial metric spaces can be obtained from the corresponding results in metric spaces.

In 2013, Ali [2] established some fixed point theorems for $\{a, b, c\}$-ctype and ntype mappings in weakly Cauchy normed spaces.

In 2017, Sharma in [21] proved fixed point theorem of contractive mapping in cone $b$ metric spaces without using the normality condition.

Different approaches and notions of $\mathrm{N}, \mathrm{N}_{\mathrm{p}}, \mathrm{N}_{\mathrm{b}}$, and F-cone metric spaces over Banach algebra have been given by Malviya et al. [19], Fernandez et al. [10], Fernandez et al. [11], and Fernandez et al. [9], respectively. Also they define generalized Lipschitz and expansive maps for such spaces and investigate some fixed points for mappings satisfying such conditions in these new frameworks.

In 2017, continuation of the generalization of cyclic weak $\phi$ of these types introduced in quasi metric spaces [3].

Definition 1.3. Suppose that $\mathfrak{C}$ is a non empty set, $\mathrm{C}$ is a cone in the Banach algebra $\mathcal{A}, \mathfrak{r} \in \mathrm{C} ; \rho(\mathfrak{r}) \geqslant 1$, and $\mathfrak{q}(\mathfrak{r})$ (simply written as $\mathfrak{q}$ ) is a function; $\mathfrak{q}: \mathfrak{C} \times \mathfrak{C} \rightarrow \mathcal{A}$; such that

1. $\Theta \ll \mathfrak{q}(u, v), \quad \forall u, v \in \mathfrak{C} ;$

2. $\mathfrak{q}(u, v)=\Theta \Longleftrightarrow u=v$;

3. $\mathfrak{q}(u, v)=\mathfrak{q}(v, u), \forall u, v \in \mathfrak{C}$;

4. $\mathfrak{q}(u, v) \leqslant$ ro $[\mathfrak{q}(u, w)+\mathfrak{q}(w, v)], \forall u, v, w \in \mathfrak{C}$.

Then $(\mathfrak{C}, C, \mathfrak{q}(\mathfrak{r}))$ is defined to be a cone quasi metric space on the Banach algebra $\mathcal{A}$. If in particular, $\mathfrak{r}=\Delta$, then $(\mathfrak{C}, \mathfrak{C}, \mathfrak{q}(\mathfrak{r}))$ simply written as $(\mathfrak{C}, \mathfrak{C}, \mathfrak{q})$ is cone metric on $\mathcal{A}$.

\subsection{Example}

Let $\mathbb{R}^{m}$ be the set of all finite $m$ sequences of real numbers, for $\left\{a_{k}\right\}_{k=1}^{m},\left\{b_{k}\right\}_{k=1}^{m}$ in $\mathbb{R}^{m}$ and $\alpha \in \mathbb{R}$. Define on $\mathbb{R}^{m}$ the following operations

$$
\alpha\left\{a_{k}\right\}_{k=1}^{m}=\left\{\alpha a_{k}\right\}_{k=1}^{m}, \quad\left\{a_{k}\right\}_{k=1}^{m}+\left\{b_{k}\right\}_{k=1}^{m}=\left\{a_{k}+b_{k}\right\}_{k=1}^{m}, \quad \text { and } \quad\left\{a_{k}\right\}_{k=1}^{m} \times\left\{b_{k}\right\}_{k=1}^{m}=\left\{a_{k} \times b_{k}\right\}_{k=1}^{m} .
$$

If $0<p \leqslant 1$, denote $\left\|\left\{a_{k}\right\}_{k=1}^{m}\right\|_{p}=\sqrt[p]{\sum_{k=1}^{m}\left|a_{k}\right|^{p}}$, we have

$$
\sqrt[p]{\sum_{k=1}^{m}\left|a_{k}+b_{k}\right|^{p}} \leqslant 2^{\frac{1}{p}}\left[\sqrt[p]{\sum_{k=1}^{m}\left|a_{k}\right| p}+\sqrt[p]{\left.\sum_{k=1}^{m}\left|b_{k}\right| p\right]}, \quad \forall\left\{a_{k}\right\}_{k=1}^{m},\left\{b_{k}\right\}_{k=1}^{m} \in \mathbb{R}^{m} .\right.
$$


Let $\mathcal{A}=\mathbf{M}\left(\mathbb{R}^{\mathrm{m}}\right)$ be the algebra of all n-square matrices whose entries are elements in $\mathbb{R}^{\mathrm{m}}$

$$
\mathcal{A}=\left\{a: a=\left\{a_{i j}\right\}_{1 \leqslant i, j \leqslant n}, a_{i j}=\left\{a_{k}^{i j}\right\}_{k=1}^{m} \in \mathbb{R}^{m}, \forall 1 \leqslant i, j \leqslant n\right\} .
$$

Operations on $\mathcal{A}$ are defined as follows:

$$
\begin{aligned}
\alpha a & =\alpha\left\{a_{i j}\right\}_{1 \leqslant i, j \leqslant n}=\left\{\alpha\left\{a_{k}^{i j}\right\}_{k=1}^{m}\right\}_{1 \leqslant i, j \leqslant n}=\left\{\left\{\alpha a_{k}^{i j}\right\}_{k=1}^{m}\right\}_{1 \leqslant i, j \leqslant n} \\
a \oplus b & =\left\{a_{i j}\right\}_{1 \leqslant i, j \leqslant n} \oplus\left\{b_{i j}\right\}_{1 \leqslant i, j \leqslant n} \\
& =\left\{a_{i j}+b_{i j}\right\}_{1 \leqslant i, j \leqslant n}=\left\{\left\{a_{k}^{i j}\right\}_{k=1}^{m}+\left\{b_{k}^{i j}\right\}_{k=1}^{m}\right\}_{1 \leqslant i, j \leqslant n}=\left\{\left\{a_{k}^{i j}+b_{k}^{i j}\right\}_{k=1}^{m}\right\}_{1 \leqslant i, j \leqslant n}
\end{aligned}
$$

and

$$
\begin{aligned}
a \odot b & =\left\{a_{i j}\right\}_{1 \leqslant i, j \leqslant n} \odot\left\{b_{i j}\right\}_{1 \leqslant i, j \leqslant n} \\
& =\left\{\sum_{l=1}^{n} a_{i l} b_{l j}\right\}_{1 \leqslant i, j \leqslant n} \\
& =\left\{\sum_{l=1}^{n}\left\{a_{k}^{i l}\right\}_{k=1} \times\left\{b_{k}^{l j}\right\}_{k=1}^{m}\right\}_{1 \leqslant i, j \leqslant n}=\left\{\sum_{l=1}^{n}\left\{a_{k}^{i l} b_{k}^{l j}\right\}_{k=1}^{m}\right\}_{1 \leqslant i, j \leqslant n}=\left\{\left\{\sum_{l=1}^{n} a_{k}^{i l} b_{k}^{l j}\right\}_{k=1}^{m}\right\}_{1 \leqslant i, j \leqslant n} .
\end{aligned}
$$

Define the norm

or

$$
\|a\|_{p}=\sum_{1 \leqslant i, j \leqslant n}\left\|\left\{a_{k}^{i j}\right\}_{k=1}^{m}\right\|_{p}=\sum_{1 \leqslant i, j \leqslant n} \sqrt[p]{\sum_{k=1}^{m}\left|a_{k}^{i j}\right| p}
$$

$$
\|a\|_{\infty}=\max _{1 \leqslant i \leqslant n} \sum_{1 \leqslant j \leqslant n}\left\|\left\{a_{k}^{i j}\right\}_{k=1}^{m}\right\|_{p}=\max _{1 \leqslant i \leqslant n} \sum_{1 \leqslant j \leqslant n} \sqrt[p]{\sum_{k=1}^{m}\left|a_{k}^{i j}\right| p .}
$$

Then $\mathcal{A}$ is a real Banach algebra, its zero element is the matrix that all of its entries are the zero element of $\mathbb{R}^{\mathrm{m}}, \mathrm{O}=\{0,0, \ldots, 0\}$. The unit element $\Delta_{\mathrm{n}}$ of $\mathcal{A}$ is the matrix whose diagonals are elements in $\mathbb{R}^{\mathrm{m}}$ each of its components is $1, e=\{1,1,1, \ldots, 1\}$ and the other entries of $\Delta_{\mathfrak{n}}$ are the zero elements in $\mathbb{R}^{\mathrm{m}}$;

$$
\Delta_{n}=\left[\begin{array}{ccccc}
e & O & O & \cdots & O \\
O & e & O & \cdots & O \\
O & O & e & \cdots & O \\
O & O & O & \cdots & O \\
O & O & O & \cdots & e
\end{array}\right]
$$

Let $C=\left\{a: a=\left\{a_{i j}\right\}_{1 \leqslant i, j \leqslant n}, a_{i j}=\left\{a_{k}^{i j}\right\}_{k=1}^{m}, 1 \leqslant i, j \leqslant n\right.$, and $a_{k}^{i j} \geqslant 0$ for all $\left.1 \leqslant i, j \leqslant n, 1 \leqslant k \leqslant m\right\}$; the set of all $n$ square matrices each entry is an m-sequence of non-negative real numbers. Then $C \subset \mathcal{A}$ is a cone.

Let $\mathfrak{C}=\mathbf{M}\left(\mathbb{R}^{\mathrm{m}}\right)$ and $\mathfrak{r}=2^{\frac{1}{\mathfrak{p}}} \Delta_{n}$. Then

$$
\begin{aligned}
\rho(\mathfrak{r})=\lim _{n \rightarrow \infty} \sqrt[n]{\left\|\left[2^{\frac{1}{p}} \Delta_{n}\right]^{n}\right\|_{p}}=\lim _{n \rightarrow \infty} \sqrt[n]{\left\|\left[2^{\frac{n}{p}} \Delta_{n}\right]\right\|_{p}} & =\lim _{n \rightarrow \infty} \sqrt[n]{\left[2^{\frac{n}{p}} n \times m^{\frac{1}{p}}\right]} \\
& =2^{\frac{1}{p}} \lim _{n \rightarrow \infty} \sqrt[n]{n \times m^{\frac{1}{p}}}=2^{\frac{1}{p}}(1)=2^{\frac{1}{p}} \geqslant 1
\end{aligned}
$$

or

$$
\rho(\mathfrak{r})=\lim _{n \rightarrow \infty} \sqrt[n]{\left\|\left[2^{\frac{1}{\mathfrak{p}}} \Delta_{n}\right]^{n}\right\|_{\infty}}=\lim _{n \rightarrow \infty} \sqrt[n]{\left\|\left[2^{\frac{n}{p}} \Delta_{n}\right]\right\|_{\infty}}=\lim _{n \rightarrow \infty} \sqrt[n]{\left[2^{\frac{n}{p}} m^{\frac{1}{p}}\right]}=2^{\frac{1}{p}} \lim _{n \rightarrow \infty} \sqrt[n]{m^{\frac{1}{p}}}=2^{\frac{1}{p}}(1)=2^{\frac{1}{p}} \geqslant 1 .
$$


Define $\mathfrak{q}: \mathfrak{C} \times \mathfrak{C} \rightarrow \mathcal{A}$ as follows:

$$
\mathfrak{q}(a, b)=\left\{\left\{\left\|a_{i j}-b_{i j}\right\|_{p} \delta_{j k}\right\}_{k=1}^{m}\right\}_{1 \leqslant i, j \leqslant n}=\left\{\left\{\sqrt[p]{\sum_{l=1}^{k}\left|a_{l}^{i j}-b_{l}^{i j}\right| p} \delta_{j k}\right\}_{k=1}^{m}\right\}_{1 \leqslant i, j \leqslant n} .
$$

Then $(\mathfrak{C}, C, \mathfrak{q}(\mathfrak{r}))$ is a cone quasi metric space on the Banach algebra $\mathcal{A}$. In fact; for any $a, b, c \in \mathfrak{C}$, we have

$$
\begin{aligned}
2^{\frac{1}{p}} \Delta_{n} \odot[\mathfrak{q}(a, c)+\mathfrak{q}(c, b)] & =2^{\frac{1}{p}} \Delta_{n} \odot\left[\left\{\left\{\left\|a_{i j}-c_{i j}\right\|_{p} \delta_{j k}\right\}_{k=1}^{m}\right\}_{1 \leqslant i, j \leqslant n}+\left\{\left\{\left\|c_{i j}-b_{i j}\right\|_{p} \delta_{j k}\right\}_{k=1}^{m}\right\}_{1 \leqslant i, j \leqslant n}\right] \\
& =2^{\frac{1}{p}} \Delta_{n} \odot\left[\left\{\left\{\left\|a_{i j}-c_{i j}\right\|_{p} \delta_{j k}\right\}_{k=1}^{m}+\left\{\left\|c_{i j}-b_{i j}\right\|_{p} \delta_{j k}\right\}_{k=1}^{m}\right\}_{1 \leqslant i, j \leqslant n}\right] \\
& =2^{\frac{1}{p}} \Delta_{n} \odot\left[\left\{\left\{\left[\left\|a_{i j}-c_{i j}\right\|_{p}+\left\|c_{i j}-b_{i j}\right\|_{p}\right] \delta_{j k}\right\}_{k=1}^{m}\right\}_{1 \leqslant i, j \leqslant n}\right] \\
& =\left\{\left\{2^{\frac{1}{p}}\left[\left\|a_{i j}-c_{i j}\right\|_{p}+\left\|c_{i j}-b_{i j}\right\|_{p}\right] \delta_{j k}\right\}_{k=1}^{m}\right\}_{1 \leqslant i, j \leqslant n} \\
& =\left\{\left\{2^{\frac{1}{p}}\left[\sqrt[p]{\sum_{l=1}^{k}\left|a_{l}^{i j}-c_{l}^{i j}\right| p}+\sqrt[p]{\left.\sum_{l=1}^{k}\left|c_{l}^{i j}-b_{l}^{i j}\right| p\right]} \delta_{j k}\right\}_{k=1}^{m}\right\}_{1 \leqslant i, j \leqslant n}\right.
\end{aligned}
$$

hence $\mathfrak{q}(a, b) \leqslant$ ro $[\mathfrak{q}(a, c)+\mathfrak{q}(c, b)], \quad \forall a, b, c \in \mathfrak{C}$ because

$$
2^{\frac{1}{p}}\left[\sqrt[p]{\sum_{l=1}^{k}\left|a_{l}^{i j}-b_{l}^{i j}\right| p}+\sqrt[p]{\left.\sum_{l=1}^{k}\left|c_{l}^{i j}-b_{l}^{i j}\right| p\right]}-\sqrt[p]{\sum_{l=1}^{k}\left|a_{l}^{i j}-b_{l}^{i j}\right| p} \geqslant 0, \forall 1 \leqslant i, j \leqslant n, 1 \leqslant k \leqslant m .\right.
$$

A sequence $\left\{v_{\mathfrak{n}}\right\}_{\mathfrak{n} \in \mathbb{N}}$ in $(\mathfrak{C}, C, \mathfrak{q}(\mathfrak{r}))$ converges to $v$ whenever for each $z \in \mathcal{A}$ with $\Theta<z$ there is $\mathfrak{n}_{0} \in \mathbb{N}$ such that $\mathfrak{q}\left(v_{n}, v\right)<z$ for all $\mathfrak{n} \geqslant n_{0}$.

A sequence $\left\{v_{\mathfrak{n}}\right\}_{\mathfrak{n} \in \mathbb{N}}$ in $(\mathfrak{C}, C, \mathfrak{q}(\mathfrak{r}))$ is Cauchy whenever for each $z \in \mathcal{A}$ with $\Theta<z$ there is $\mathfrak{n}_{0} \in \mathbb{N}$ such that $\mathfrak{q}\left(v_{n}, v_{m}\right)<z$ for all $n, m \geqslant n_{0}$.

A cone quasi metric space on a Banach algebra $(\mathfrak{C}, C, \mathfrak{q}(\mathfrak{r}))$ is complete whenever every Cauchy sequence in $\mathfrak{C}$ converges to an element belonging to $\mathfrak{C}$.

Also in [18] the convergence and Cauchy-ness of sequences is defined in terms of the relation $\ll$. Denoting this convergence by $v_{n} \rightarrow^{C} v$, it follows:

$$
v_{\mathrm{n}} \rightarrow^{\mathrm{C}} v \Longleftrightarrow \mathfrak{q}\left(v_{\mathrm{n}}, v\right) \rightarrow \Theta \quad(\text { in } \mathcal{A}),
$$

provided the cone $\mathrm{C}$ is normal (another hypothesis assumed in [18]).

In this paper we introduce the following concepts.

Definition 1.4. Let $(\mathfrak{C}, C, \mathfrak{q})$ be a cone metric on a Banach algebra $\mathcal{A}$ and $S$ be a self mapping on $\mathfrak{C}$. If there are vectors $x, y, z \in \mathcal{A}$ with spectral radius $\rho\left[(\Delta-y)^{-1}(x+z)\right]<1$ such that

$$
\mathfrak{q}(\mathrm{S}(\mathrm{u}), \mathrm{S}(v)) \ll x o \mathfrak{q}(u, v)+\text { yo } \mathfrak{q}(v, S(v))+z o \mathfrak{q}(u, S(u)), \quad \forall u, v \in \mathfrak{C},
$$

then $\mathrm{S}$ is said to be generalized Banach algebra contraction on $\mathfrak{C}$.

We have the following.

Remark 1.5. If $\mathrm{a}, \mathrm{b}, \mathrm{c}$ are scalars, $\mathrm{a}, \mathrm{b}, \mathrm{c} \in[0,1), \mathrm{a}+\mathrm{b}+\mathrm{c}<1, \mathrm{x}=\mathrm{a} \Delta, \mathrm{y}=\mathrm{b} \Delta, z=\mathrm{c} \Delta \in \mathcal{A}$, then scalarization metric function insures that $S$ is reduced to the classical case of abc-generalized contraction types of mappings, see [2, 3, 8].

More general concept is as follows.

Definition 1.6. Let $(\mathfrak{C}, C, \mathfrak{q}(\mathfrak{r}))$ be a cone quasi metric on a Banach algebra $\mathcal{A}, \emptyset \neq A \subset \mathfrak{C}$ and $\emptyset \neq B \subset \mathfrak{C}$, $\mathfrak{C}=A \cup B$, and $S$ be a self mapping on $\mathfrak{C}$. Then $S$ is generalized cyclic Banach algebra contraction on $\mathfrak{C}$ with respect to the pair $(A, B)$ iff $S$ fulfils the following: 
1. $\mathrm{S}(\mathrm{A}) \subset \mathrm{B}$ and $\mathrm{S}(\mathrm{B}) \subset \mathrm{A}$; and

2. there are vectors $x, y, z \in \mathcal{A}$ with $\Theta \ll x, y, z, \rho(x)<1, \rho(y)<1, \rho\left(\right.$ ro $\left.(\Delta-y)^{-1} 0(x+z)\right)<1$, and

$$
\mathfrak{q}(S(u), S(v)) \ll x o \mathfrak{q}(u, v)+\operatorname{yoq}(v, S(v))+z o \mathfrak{q}(u, S(u)), \quad \forall u \in A, v \in B .
$$

Remark 1.7.

1. For cyclical concepts in classical case, see [17].

2. Any generalized Banach algebra contraction on $\mathfrak{C}$ is cyclic generalized (with $\mathfrak{r}=\Delta$ ) Banach algebra contraction on $\mathfrak{C}$ with respect to the pair $(\mathfrak{C}, \mathfrak{C})$.

Through this work $\mathfrak{C}$ is $C-\mathfrak{q}(\mathfrak{r})$ cone quasi metric space in the Banach algebra $\mathcal{A}, A \neq \emptyset$ and $B \neq \emptyset$ in $\mathfrak{C}$, $\mathfrak{C}=A \cup B, S$ and $T$ are mappings from $\mathfrak{C}$ into $\mathfrak{C}$, and for simplicity we will drop the o of the multiplication operation of the Banach algebra $\mathcal{A}$.

In this paper, generalization of the contraction and Kannan types of mappings on wider class of spaces; (namely $\mathrm{C}-\mathfrak{q}(\mathfrak{r})$ cone quasi metric spaces on Banach algebra) are given. Next; generalized cyclic Banach algebra generalized contractions are introduced. The existence of a unique common fixed point of two self mappings of such types is proved, and then fixed point theorem of generalized cyclic Banach algebra contractions on $C-\mathfrak{q}(\mathfrak{r})$ cone quasi metric space $\mathfrak{C}$ with respect to a couple of compact sets $(A, B)$ is established. Consequently; generalization of the classical concepts are given and new fixed point results are proved.

\section{Main results}

We have the following theorem.

Theorem 2.1. Let $(\mathfrak{C}, C, \mathfrak{q}(\mathfrak{r}))$ be a cone quasi metric space in a Banach algebra $\mathcal{A}$ and $\left\{v_{\mathfrak{n}}\right\}_{\mathfrak{n} \in \mathrm{N}}$ be in $\mathfrak{C}$ such that

$$
\mathfrak{q}\left(v_{\mathrm{n}+2}, v_{\mathrm{n}+1}\right) \ll \mathfrak{r}_{\mathfrak{o}} \mathfrak{q}\left(v_{\mathrm{n}+1}, v_{\mathrm{n}}\right), \quad \mathrm{n}=0,1,2, \ldots
$$

for some vector $\mathfrak{r}_{\mathfrak{o}} \in \mathrm{C}$ which commutes with $\mathfrak{r}$ and $\rho(\mathfrak{r}) \rho\left(\mathfrak{r}_{\mathfrak{o}}\right)<1$.Then $\left\{v_{n}\right\}_{\mathfrak{n} \in \mathrm{N}}$ is Cauchy.

Proof. Taking into account the conditions $\mathrm{C}^{2} \subset \mathrm{C}$ and $\mathrm{C}+\mathrm{C} \subset \mathrm{C}$, it follows that

$$
\mathfrak{q}\left(v_{n+1}, v_{n}\right) \ll \mathfrak{r}_{\mathfrak{o}} \mathfrak{q}\left(v_{n}, v_{n-1}\right),
$$

this implies,

$$
\mathfrak{r}_{\mathfrak{o}} \mathfrak{q}\left(v_{\mathrm{n}}, v_{\mathrm{n}-1}\right)-\mathfrak{q}\left(v_{\mathrm{n}+1}, v_{\mathfrak{n}}\right) \in \mathrm{C}
$$

Since $\mathfrak{r}_{\mathfrak{o}} \in C$, we have

$$
\mathfrak{r}_{\mathfrak{o}}\left[\mathfrak{r}_{\mathfrak{o}} \mathfrak{q}\left(v_{n}, v_{n-1}\right)-\mathfrak{q}\left(v_{n+1}, v_{n}\right)\right] \in C,
$$

that is

$$
\mathfrak{r}_{\mathfrak{o}} \mathfrak{r}_{\mathfrak{o}} \mathfrak{q}\left(v_{n}, v_{n-1}\right)-\mathfrak{r}_{\mathfrak{o}} \mathfrak{q}\left(v_{n+1}, v_{\mathfrak{n}}\right) \in \mathrm{C}
$$

Consequently

$$
\mathfrak{r}_{\mathfrak{o}}^{2} \mathfrak{q}\left(v_{n}, v_{n-1}\right)-\mathfrak{r}_{\mathfrak{o}} \mathfrak{q}\left(v_{n+1}, v_{\mathfrak{n}}\right) \in \mathrm{C}
$$

Additionally, $\mathfrak{q}\left(v_{\mathfrak{n}+2}, v_{\mathfrak{n}+1}\right) \ll \mathfrak{r}_{\mathfrak{o}} \mathfrak{q}\left(v_{n+1}, v_{\mathfrak{n}}\right)$ gives

$$
\mathfrak{r}_{\mathfrak{o}} \mathfrak{q}\left(v_{\mathrm{n}+1}, v_{\mathrm{n}}\right)-\mathfrak{q}\left(v_{\mathrm{n}+2}, v_{\mathrm{n}+1}\right) \in \mathrm{C} .
$$

Inclusions (2.1) and (2.2) yield the following:

$$
\left[\mathfrak{r}_{\mathfrak{o}}^{2} \mathfrak{q}\left(v_{n}, v_{n-1}\right)-\mathfrak{r}_{\mathfrak{o}} \mathfrak{q}\left(v_{n+1}, v_{n}\right)\right]+\left[\mathfrak{r}_{\mathfrak{o}} \mathfrak{q}\left(v_{n+1}, v_{n}\right)-\mathfrak{q}\left(v_{n+2}, v_{n+1}\right)\right] \in \mathbf{C},
$$


thus

$$
\left[\mathfrak{r}_{\mathfrak{o}}^{2} \mathfrak{q}\left(v_{n}, v_{n-1}\right)-\mathfrak{q}\left(v_{n+2}, v_{n+1}\right)\right] \in \mathrm{C},
$$

hence

$$
\mathfrak{q}\left(v_{n+2}, v_{n+1}\right) \ll \mathfrak{r}_{\mathfrak{o}} \mathfrak{q}\left(v_{n+1}, v_{n}\right) \ll \mathfrak{r}_{\mathfrak{o}}^{2} \mathfrak{q}\left(v_{n}, v_{n-1}\right)
$$

This implies successively,

$$
\mathfrak{q}\left(v_{n}, v_{\mathfrak{n}+1}\right) \ll \mathfrak{r}_{\mathfrak{o}} \mathfrak{q}\left(v_{n-1}, v_{n}\right) \ll \mathfrak{r}_{\mathfrak{o}}^{2} \mathfrak{q}\left(v_{n-2}, v_{n-1}\right) \ll \cdots \ll \mathfrak{r}_{\mathfrak{o}}{ }^{n} \mathfrak{q}\left(v_{0}, v_{1}\right) .
$$

Now let $n, m \in \mathbb{N}$ be such that $m>n$ and use the transitive and positivity properties of $C$. We have

$$
\begin{aligned}
\mathfrak{q}\left(v_{\mathfrak{n}}, v_{\mathfrak{m}}\right) & \ll \mathfrak{r}\left[\mathfrak{q}\left(v_{n}, v_{\mathfrak{n}+1}\right)+\mathfrak{q}\left(v_{\mathfrak{n}+1}, v_{\mathfrak{m}}\right)\right] \\
& \ll \mathfrak{r}\left[\mathfrak{q}\left(v_{n}, v_{\mathfrak{n}+1}\right)+\mathfrak{r}\left\{\mathfrak{q}\left(v_{\mathfrak{n}+1}, v_{\mathfrak{n}+2}\right)+\mathfrak{q}\left(v_{\mathfrak{n}+2}, v_{\mathfrak{m}}\right)\right\}\right] \\
& \ll \mathfrak{r} \mathfrak{q}\left(v_{\mathfrak{n}}, v_{\mathfrak{n}+1}\right)+\mathfrak{r}^{2} \mathfrak{q}\left(v_{\mathfrak{n}+1}, v_{\mathfrak{n}+2}\right)+\mathfrak{r}^{2}\left[\mathfrak{r}\left\{\mathfrak{q}\left(v_{\mathfrak{n}+2}, v_{\mathfrak{n}+3}\right)+\mathfrak{q}\left(v_{\mathfrak{n}+3}, v_{\mathfrak{m}}\right)\right\}\right] \\
& \ll \mathfrak{r} \mathfrak{q}\left(v_{n}, v_{n+1}\right)+\mathfrak{r}^{2} \mathfrak{q}\left(v_{n+1}, v_{\mathfrak{n}+2}\right)+\mathfrak{r}^{3} \mathfrak{q}\left(v_{\mathfrak{n}+2}, v_{\mathfrak{n}+3}\right)+\cdots+\mathfrak{r}^{\mathfrak{m}-\mathfrak{n}} \mathfrak{q}\left(v_{m-1}, v_{m}\right) .
\end{aligned}
$$

Back to inequalities (2.3), we get

$$
\begin{aligned}
& \mathfrak{q}\left(v_{n}, v_{m}\right) \ll \mathfrak{r r}_{\mathfrak{o}}{ }^{n} \mathfrak{q}\left(v_{1}, v_{0}\right)+\mathfrak{r}^{2} \mathfrak{r}_{\mathfrak{o}}{ }^{n+1} \mathfrak{q}\left(v_{1}, v_{0}\right)+\mathfrak{r}^{3} \mathfrak{r}_{\mathfrak{o}}{ }^{n+2} \mathfrak{q}\left(v_{1}, v_{0}\right)+\cdots+\mathfrak{r}^{\mathfrak{m}-n} \mathfrak{r}_{\mathfrak{o}}{ }^{n+(m-1-n)} \mathfrak{q}\left(v_{1}, v_{0}\right) \\
& \ll\left[\mathfrak{r r}_{\mathfrak{o}}{ }^{n}+\mathfrak{r}^{2} \mathfrak{r}_{\mathfrak{o}}{ }^{n+1}+\mathfrak{r}^{3} \mathfrak{r}_{\mathfrak{o}}{ }^{n+2}+\cdots+\mathfrak{r}^{m-n} \mathfrak{r}_{\mathfrak{o}}{ }^{n+(m-1-n)}\right] \mathfrak{q}\left(v_{1}, v_{0}\right) \\
& =\mathfrak{r}\left[\Delta+\mathfrak{r r}_{\mathfrak{o}}+\left(\mathfrak{r r}_{\mathfrak{o}}\right)^{2}+\cdots+\left(\mathfrak{r} \mathfrak{r}_{\mathfrak{o}}\right)^{(\mathfrak{m}-1-\mathfrak{n})}\right] \mathfrak{r}_{\mathfrak{o}}{ }^{n} \mathfrak{q}\left(v_{1}, v_{0}\right),
\end{aligned}
$$

we see that

$$
\mathfrak{r}\left[\Delta+\mathfrak{r r}_{\mathfrak{o}}+\left(\mathfrak{\mathfrak { r r } _ { \mathfrak { o } }}\right)^{2}+\cdots+\left(\mathfrak{r r}_{\mathfrak{o}}\right)^{(\mathfrak{m}-1-\mathfrak{n})}\right] \mathfrak{r}_{\mathfrak{o}} \mathfrak{n}_{\mathfrak{q}}\left(v_{1}, v_{0}\right)-\mathfrak{q}\left(v_{\mathfrak{n}}, v_{\mathfrak{m}}\right) \in \mathrm{C}
$$

Since

$$
\mathfrak{r}\left(\mathfrak{r} \mathfrak{r}_{\mathfrak{o}}\right)^{(m-1-n+j)} \mathfrak{r}_{\mathfrak{o}}{ }^{n} \mathfrak{q}\left(v_{1}, v_{0}\right) \in C, \quad \forall \mathfrak{j} \in \mathbb{N}
$$

we have

$\mathfrak{r}\left(\mathfrak{r r}_{\mathfrak{o}}\right)^{(\mathfrak{m}-1-\mathfrak{n}+\mathfrak{j})} \mathfrak{r}_{\mathfrak{o}}{ }^{\mathfrak{q}} \mathfrak{q}\left(v_{1}, v_{0}\right)+\left[\mathfrak{r}\left[\Delta+\mathfrak{r} \mathfrak{r}_{\mathfrak{o}}+\left(\mathfrak{r} \mathfrak{r}_{\mathfrak{o}}\right)^{2}+\cdots+\left(\mathfrak{r} \mathfrak{r}_{\mathfrak{o}}\right)^{(\mathfrak{m}-1-\mathfrak{n})}\right] \mathfrak{r}_{\mathfrak{o}}{ }^{\mathfrak{n}} \mathfrak{q}\left(v_{1}, v_{0}\right)-\mathfrak{q}\left(v_{\mathfrak{n}}, v_{\mathfrak{m}}\right)\right] \in \mathrm{C}, \forall \mathfrak{j} \in \mathbb{N}$, that is

$$
\begin{aligned}
& {\left[\mathfrak{r}\left[\Delta+\mathfrak{n} \mathfrak{r}_{\mathfrak{o}}+\left(\mathfrak{n} \mathfrak{r}_{\mathfrak{o}}\right)^{2}+\cdots+\left(\mathfrak{n} \mathfrak{r}_{\mathfrak{o}}\right)^{(m-1-\mathfrak{n})}\right] \mathfrak{r}_{\mathfrak{o}} \mathfrak{n}_{\mathfrak{q}}\left(v_{1}, v_{0}\right)\right.} \\
& \left.+\mathfrak{r}\left(\mathfrak{r r}_{\mathfrak{o}}\right)^{(\mathrm{m}-1-\mathfrak{n}+\mathfrak{j})} \mathfrak{r}_{\mathfrak{o}}{ }^{n} \mathfrak{q}\left(v_{1}, v_{0}\right)\right]-\mathfrak{q}\left(v_{\mathfrak{n}}, v_{\mathfrak{m}}\right) \in \mathrm{C}, \quad \forall \mathfrak{j} \in \mathbb{N} .
\end{aligned}
$$

Equivalently

$$
\begin{aligned}
\mathfrak{r}[\Delta+ & \mathfrak{r r}_{\mathfrak{o}}+\left(\mathfrak{r r}_{\mathfrak{o}}\right)^{2}+\cdots+\left(\mathfrak{r} \mathfrak{r}_{\mathfrak{o}}\right)^{(\mathfrak{m}-1-\mathfrak{n})} \\
& \left.+\left(\mathfrak{r r}_{\mathfrak{o}}\right)^{(\mathfrak{m}-1-\mathfrak{n}+\mathfrak{j})}\right] \mathfrak{r}_{\mathfrak{o}}{ }^{n} \mathfrak{q}\left(v_{1}, v_{0}\right)-\mathfrak{q}\left(v_{\mathfrak{n}}, v_{\mathfrak{m}}\right) \in \mathrm{C}, \quad \forall \mathfrak{j} \in \mathbb{N} .
\end{aligned}
$$

Continuing this process shows that

$$
\mathfrak{q}\left(v_{\mathfrak{n}}, v_{\mathfrak{m}}\right) \ll \mathfrak{r}\left[\Delta+\mathfrak{r} \mathfrak{r}_{\mathfrak{o}}+\left(\mathfrak{r} \mathfrak{r}_{\mathfrak{o}}\right)^{2}+\cdots\right] \mathfrak{r}_{\mathfrak{o}} \mathfrak{n}_{\mathfrak{q}}\left(v_{1}, v_{0}\right) .
$$

Since $\rho(\mathfrak{r}) \rho\left(\mathfrak{r}_{\mathfrak{o}}\right)<1$, we have $\rho\left(\mathfrak{r} \mathfrak{r}_{\mathfrak{o}}\right)<1$, hence the element $\left(\Delta-\mathfrak{r} \mathfrak{r}_{\mathfrak{o}}\right)$ is invertible and the series $\sum_{n=1}^{\infty}\left(\mathfrak{r r}_{\mathfrak{o}}\right)^{n-1}$ is convergent. Moreover

$$
\left(\Delta-\mathfrak{r r}_{\mathfrak{o}}\right)^{-1}=\sum_{n=1}^{\infty}\left(\mathfrak{r r}_{\mathfrak{o}}\right)^{\mathfrak{n}-1},
$$


substituting in (2.4) gives

$$
\mathfrak{q}\left(v_{n}, v_{\mathfrak{m}}\right) \ll \mathfrak{r}\left[\sum_{\mathfrak{n}=1}^{\infty}\left(\mathfrak{r} \mathfrak{r}_{\mathfrak{o}}\right)^{\mathfrak{n}-1}\right] \mathfrak{r}_{\mathfrak{o}}{ }^{n} \mathfrak{q}\left(v_{1}, v_{0}\right) \ll \mathfrak{r}\left(\Delta-\mathfrak{r} \mathfrak{r}_{\mathfrak{o}}\right)^{-1} \mathfrak{r}_{\mathfrak{o}}{ }^{n} \mathfrak{q}\left(v_{1}, v_{0}\right) .
$$

Since $\rho(\mathfrak{r}) \geqslant 1$ and $\rho(\mathfrak{r}) \rho\left(\mathfrak{r}_{\mathfrak{o}}\right)<1$, we have $\rho\left(\mathfrak{r}_{\mathfrak{o}}\right)<1$, hence $\left\{\mathfrak{r}_{\mathfrak{o}}{ }^{n}\right\}_{\mathfrak{n} \in N}$ is absolutely convergent to $\Theta$ (because $\left\{\mathfrak{r}_{\mathfrak{o}}{ }^{n}\right\}_{\mathfrak{n} \in N}$ are the $n^{\prime}$ s terms of a norm convergent series $\sum_{n=1}^{\infty} \mathfrak{r}_{\mathfrak{o}}{ }^{n-1}$ ), taking the limit as $n \rightarrow \infty$ of the two sides of (2.5) shows that $\mathfrak{r}\left(\Delta-\mathfrak{r r}_{\mathfrak{o}}\right)^{-1} \mathfrak{r}_{\mathfrak{o}}{ }^{n} \mathfrak{q}\left(v_{1}, v_{0}\right) \rightarrow \Theta$ as $n \rightarrow \infty$ and completes the proof.

We have the following theorem.

Theorem 2.2. Let $\mathfrak{C}$ be a complete cone quasi metric space, $\mathfrak{C}=A \cup B, S, T: \mathfrak{C} \rightarrow \mathfrak{C}$ be two mappings such that $\mathrm{S}(\mathrm{A}) \subset \mathrm{B}, \mathrm{T}(\mathrm{B}) \subset \mathrm{A}$, and

$$
\mathfrak{q}(\mathrm{S}(\mathrm{u}), \mathrm{T}(v)) \ll x \mathfrak{q}(u, v)+y \mathfrak{q}(u, S(u))+z \mathfrak{q}(v, \mathrm{~T}(v)), \quad \forall \mathfrak{u} \in A, v \in B,
$$

where $x, y, z \in \mathcal{A}, \mathfrak{r}[\Delta-y]^{-1}(x+z)=[\Delta-y]^{-1}(x+z) \mathfrak{r}$ with $\Theta \ll x, y, z$ and $\rho(\mathfrak{r}) \rho\left([\Delta-y]^{-1}(x+z)\right)<1$. Then there is a single point $w \in \mathfrak{C}$ such that $\mathrm{S}(w)=\mathrm{T}(w)=w \in A \cap \mathrm{B}$.

Proof. Let $\mathfrak{u}_{0}$ be an arbitrarily chosen element in $\mathfrak{C}$, if $\mathfrak{u}_{0} \in B$, then $u_{1}=T\left(u_{0}\right) \in A, u_{2}=S\left(u_{1}\right) \in B$, $u_{3}=T\left(u_{2}\right) \in A$, and then define by induction:

$$
\mathrm{u}_{2 \mathrm{n}+2}=\mathrm{S}\left(\mathrm{u}_{2 \mathrm{n}+1}\right) \in \mathrm{B} \text { and } \mathrm{u}_{2 \mathrm{n}+1}=\mathrm{T}\left(\mathrm{u}_{2 \mathrm{n}}\right) \in A, \quad \forall \mathrm{n} \geqslant 0 .
$$

First, suppose $n$ is an odd natural number. Then

$$
\begin{aligned}
\mathfrak{q}\left(u_{n+1}, u_{n}\right)=\mathfrak{q}\left(S\left(u_{n}\right), T\left(u_{n-1}\right)\right) & \ll x \mathfrak{q}\left(u_{n}, u_{n-1}\right)+y \mathfrak{q}\left(S\left(u_{n}\right), u_{n}\right)+z \mathfrak{q}\left(T\left(u_{n-1}\right), u_{n-1}\right) \\
& =x \mathfrak{q}\left(u_{n}, u_{n-1}\right)+y \mathfrak{q}\left(u_{n+1}, u_{n}\right)+z \mathfrak{q}\left(u_{n}, u_{n-1}\right) \\
& =(x+z) \mathfrak{q}\left(u_{n}, u_{n-1}\right)+y \mathfrak{q}\left(u_{n+1}, u_{n}\right)
\end{aligned}
$$

thus

$$
\mathfrak{q}\left(u_{n+1}, u_{n}\right) \ll\left[(\Delta-y)^{-1}(x+z)\right] \mathfrak{q}\left(u_{n}, u_{n-1}\right) .
$$

Second; by a similar method when $n$ is an even natural number, we obtain the same conclusion, taking $\mathfrak{r}_{\mathfrak{o}}=(\Delta-y)^{-1}(x+z)$ and using Theorem (2.1) prove that the sequence $\left\{u_{n}\right\}_{\mathfrak{n} \in N}$ is Cauchy, the completeness of $\mathfrak{C}$ insures the existence of $w_{0} \in \mathfrak{C}$ such that $\lim _{\mathfrak{n} \rightarrow \infty} \mathfrak{u}_{\mathfrak{n}}=w_{0}$. Since any subsequence of $\left\{\mathfrak{u}_{\mathfrak{n}}\right\}_{\mathfrak{n} \in N}$ should converge to the same limit $w_{0}$, then

$$
\lim _{n \rightarrow \infty} u_{2 n+2}=w_{0} \text { and } \lim _{n \rightarrow \infty} u_{2 n+1}=w_{0} .
$$

That is

$$
\lim _{n \rightarrow \infty} S\left(u_{2 n+1}\right)=w_{0} \text { and } \lim _{n \rightarrow \infty} T\left(u_{2 n}\right)=w_{0}
$$

Now

$$
\begin{aligned}
& \mathfrak{q}\left(\mathfrak{u}_{2 \mathfrak{n}+2}, \mathbf{T}\left(w_{0}\right)\right)=\mathfrak{q}\left(S\left(\mathfrak{u}_{2 \mathfrak{n}+1}\right), \mathbf{T}\left(w_{0}\right)\right) \\
& \ll x \mathfrak{q}\left(u_{2 n+1}, w_{0}\right)+y \mathfrak{q}\left(S\left(u_{2 n+1}\right), u_{2 n+1}\right)+z \mathfrak{q}\left(T\left(w_{0}\right), w_{0}\right) \\
& \ll x \mathfrak{q}\left(u_{2 n+1}, w_{0}\right)+y \mathfrak{q}\left(u_{2 n+2}, u_{2 n+1}\right)+z \mathfrak{q}\left(T\left(w_{0}\right), w_{0}\right) .
\end{aligned}
$$

On limiting approach as $n \rightarrow \infty$, equation (2.6) sufficiently proves that:

$$
\mathfrak{q}\left(w_{0}, \mathrm{~T}\left(w_{0}\right)\right) \ll z \mathfrak{q}\left(\mathrm{T}\left(w_{0}\right), w_{0}\right),
$$

hence $(\Delta-z) \mathfrak{q}\left(w_{0}, T\left(w_{0}\right)\right) \ll \Theta$, multiplying the two sides with $(\Delta-z)^{-1}$, we get $\mathfrak{q}\left(w_{0}, \boldsymbol{T}\left(w_{0}\right)\right)=\Theta$, that is $T\left(w_{0}\right)=w_{0}$ and $w_{0}$ is a fixed point of $T$. Similarly, we prove that $S\left(w_{0}\right)=w_{0}$. 
If there exists another point $w$ such that $S(w)=w=T(w)$, then

$$
\mathfrak{q}\left(w_{0}, w\right)=\mathfrak{q}\left(S\left(w_{0}\right), T(w)\right) \ll x \mathfrak{q}\left(w_{0}, w\right)+\mathfrak{y} \mathfrak{q}\left(S\left(w_{0}\right), w_{0}\right)+z \mathfrak{q}(\mathrm{T}(w), w)=x \mathfrak{q}\left(w_{0}, w\right)
$$

This shows that $(\Delta-x) \mathfrak{q}\left(w_{0}, w\right) \ll \Theta$, multiplying the two sides with $(\Delta-x)^{-1}$ gives $\mathfrak{q}\left(w_{0}, w\right) \ll \Theta$, that is $\Theta-\mathfrak{q}\left(w_{0}, w\right) \in C$ or $\mathfrak{q}\left(w_{0}, w\right) \in-C$, but $\mathfrak{q}\left(w_{0}, w\right) \in C$, hence $\mathfrak{q}\left(w_{0}, w\right) \in-C \cap C=\{\Theta\}$, this shows that $\mathfrak{q}\left(w_{0}, w\right)=\Theta$, consequently $w_{0}=w$.

Corollary 2.3. If $S$ is a generalized cyclic Banach algebra contraction on a complete cone quasi metric space $\mathfrak{C}$ with respect to $(\mathrm{A}, \mathrm{B})$, then $\mathrm{S}$ has only one fixed point.

For the Kannan type of mappings, we first prove the following.

Lemma 2.4. If $\mathfrak{y}_{\mathfrak{O}} \in \mathcal{A}$ with $\rho\left(\mathfrak{y}_{\mathfrak{o}}\right)<\frac{1}{2}$, then $\rho\left(\left[\Delta-\mathfrak{y}_{\mathcal{o}}\right]^{-1} \mathfrak{y}_{\mathcal{O}}\right) \leqslant \frac{1}{2}$. Moreover

$$
\left\{\Delta-\left[\Delta-\mathfrak{y}_{\mathfrak{o}}\right]^{-1} \mathfrak{y}_{\mathfrak{o}}\right\}^{-1}=\Sigma_{\mathfrak{n}=1}^{\infty}\left[\Delta-\mathfrak{y}_{\mathfrak{o}}\right]^{1-n_{\mathfrak{y}_{\mathfrak{o}}}}{ }^{\mathfrak{n}-1} .
$$

Proof. Taking in account that $\rho\left(\mathfrak{y}_{\mathfrak{o}}\right)=\lim _{\mathfrak{n} \rightarrow \infty} \sqrt[\mathfrak{n}]{\left\|\mathfrak{y}_{\mathfrak{o}}^{\mathfrak{n}}\right\|}<\frac{1}{2}$, we have $\frac{1}{2}-\rho\left(\mathfrak{y}_{\mathfrak{o}}\right)>0$, hence for $\epsilon_{0}=$ $\frac{1}{2}-\rho\left(\mathfrak{y}_{\mathfrak{o}}\right)$ there exists a natural number $n_{0}$ such that

$$
\left|\sqrt[\mathfrak{n}]{\left\|\mathfrak{y}_{\mathfrak{o}}^{\mathfrak{n}}\right\|}-\rho\left(\mathfrak{y}_{\mathfrak{o}}\right)\right|<\epsilon_{0}, \quad \forall \mathfrak{n} \geqslant \mathfrak{n}_{0}, \quad-\epsilon_{0}+\rho\left(\mathfrak{y}_{\mathfrak{o}}\right)<\sqrt[\mathfrak{n}]{\left\|\mathfrak{y}_{\mathfrak{o}}^{\mathfrak{n}}\right\|}<\rho\left(\mathfrak{y}_{\mathfrak{o}}\right)+\epsilon_{0}=\frac{1}{2}, \quad \forall \mathfrak{n} \geqslant \mathfrak{n}_{0},
$$

hence

$$
\sqrt[n_{0}+j]{\left\|\mathfrak{y}_{\mathcal{O}} \mathfrak{n}_{0}+\mathfrak{j}\right\|}<\frac{1}{2}, \forall j \geqslant 0
$$

since

$$
\left[\Delta-\mathfrak{y}_{\mathfrak{o}}\right]^{-1}=\Sigma_{n=1}^{\infty} \mathfrak{y}_{\mathfrak{o}}{ }^{(n-1)},
$$

both $\left[\Delta-\mathfrak{y}_{\mathfrak{o}}\right]^{-1}$ and $\mathfrak{y}_{\mathfrak{o}}$ are commuting, $\left(\left[\Delta-\mathfrak{y}_{\mathfrak{o}}\right]^{-1} \mathfrak{y}_{\mathfrak{o}}\right)^{\mathfrak{n}_{0}}=\left[\Delta-\mathfrak{y}_{\mathfrak{o}}\right]^{-\mathfrak{n}_{0}} \mathfrak{y}_{\mathfrak{o}}{ }^{\mathfrak{n}_{0}}$. Denote

$$
\left(\left[\Delta-\mathfrak{y}_{\mathfrak{o}}\right]^{-1}\right)^{\mathfrak{n}_{0}}=\left(\sum_{j=1}^{\infty} \mathfrak{y}_{\mathfrak{o}}{ }^{(j-1)}\right)^{\mathfrak{n}_{0}}=\sum_{j=1}^{\infty} \gamma_{j}^{\mathfrak{n}_{0}} \mathfrak{y}_{\mathcal{o}}{ }^{j} .
$$

We have

$$
\begin{aligned}
& \left\|\left(\left[\Delta-\mathfrak{y}_{\mathfrak{o}}\right]^{-1} \mathfrak{y}_{\mathfrak{o}}\right)^{\mathfrak{n}_{0}}\right\|=\left\|\left[\Delta-\mathfrak{y}_{\mathfrak{o}}\right]^{-\mathfrak{n}_{0}} \mathfrak{y}_{\mathfrak{o}}{ }^{\mathfrak{n}_{0}}\right\|=\left\|\Sigma_{j=1}^{\infty} \gamma_{j}^{\mathfrak{n}_{0}} \mathfrak{y}_{\mathfrak{o}} \mathfrak{y}_{\mathfrak{o}}{ }^{\mathfrak{n}_{0}}\right\| \\
& =\left\|\Sigma_{j=1}^{\infty} \gamma_{j}^{\mathfrak{n}_{0}} \mathfrak{y}_{\mathcal{o}}{ }^{\left(j+n_{0}\right)}\right\| \leqslant \Sigma_{j=1}^{\infty} \gamma_{j}^{\mathfrak{n}_{0}}\left\|\mathfrak{y}_{\mathcal{o}}{ }^{\left(j+n_{0}\right)}\right\| \leqslant \Sigma_{j=1}^{\infty} \gamma_{j}^{\mathfrak{n}_{0}}\left[\frac{1}{2}\right]^{\left(j+n_{0}\right)} \\
& =\left[\frac{1}{2}\right]^{n_{0}} \sum_{j=1}^{\infty} \gamma_{j}^{n_{0}}\left[\frac{1}{2}\right]^{j}=\left[\frac{1}{2}\right]^{n_{0}}\left(\sum_{j=1}^{\infty}\left[\frac{1}{2}\right]^{(j-1)}\right)^{n_{0}}=\left[\frac{1}{2}\right]^{n_{0}}\left[\frac{\frac{1}{2}}{1-\frac{1}{2}}\right]^{n_{0}}=\left[\frac{1}{2}\right]^{n_{0}} .
\end{aligned}
$$

Thus $\sqrt[n_{0}]{\left\|\left(\left[\Delta-\mathfrak{y}_{\mathfrak{o}}\right]^{-1} \mathfrak{y}_{\mathfrak{o}}\right)^{\mathfrak{n}_{0}}\right\|} \leqslant \frac{1}{2}$, consequently, we have

$$
\lim _{n \rightarrow \infty} \sqrt[n]{\left\|\left(\left[\Delta-\mathfrak{y}_{\mathfrak{o}}\right]^{-1} \mathfrak{y}_{\mathfrak{o}}\right)^{\mathfrak{n}}\right\|}=\inf _{\mathfrak{n} \in \mathbb{N}} \sqrt[n]{\left\|\left(\left[\Delta-\mathfrak{y}_{\mathfrak{o}}\right]^{-1} \mathfrak{y}_{\mathfrak{o}}\right)^{\mathfrak{n}}\right\|} \leqslant \sqrt[n_{0}]{\left\|\left(\left[\Delta-\mathfrak{y}_{\mathfrak{o}}\right]^{-1} \mathfrak{y}_{\mathfrak{o}}\right)^{\mathfrak{n}_{0}}\right\|} \leqslant \frac{1}{2}
$$

We have the following.

Definition 2.5. Let $(\mathfrak{C}, C, \mathfrak{q}(\mathfrak{r}))$ be a cone quasi metric on a Banach algebra $\mathcal{A}, \emptyset \neq A \subset \mathfrak{C}$ and $\emptyset \neq B \subset \mathfrak{C}$, $\mathfrak{C}=A \cup B$, and $S$ a self mapping on $\mathfrak{C}$. Then $S$ is said to be cyclic Banach algebra Kannan type on $\mathfrak{C}$ with respect to the pair $(A, B)$ iff $S$ fulfills the following:

1. $S(A) \subset B$ and $S(B) \subset A$; and 
2. there is vector $x \in \mathcal{A}$ with $\Theta \ll x, \rho(x \mathfrak{r})<\frac{1}{2}$, and

$$
\mathfrak{q}(\mathrm{S}(\mathrm{u}), \mathrm{S}(v)) \ll x[\mathfrak{q}(u, S(v))+\mathfrak{q}(v, \mathrm{~S}(\mathrm{u}))], \quad \forall \mathfrak{u} \in A, v \in B .
$$

Theorem 2.6. Let $(\mathfrak{C}, C, \mathfrak{q}(\mathfrak{r}))$ be a cone quasi metric on a Banach algebra $\mathcal{A}, \emptyset \neq A \subset \mathfrak{C}$ and $\emptyset \neq B \subset \mathfrak{C}$, $\mathfrak{C}=A \cup B, S$ and $\mathrm{T}$ be a self mappings on $\mathfrak{C}$ with the following:

1. $\mathrm{S}(\mathrm{A}) \subset \mathrm{B}$ and $\mathrm{T}(\mathrm{B}) \subset \mathrm{A}$; and

2. there is a vector $\mathrm{x} \in \mathcal{A}$ with $\Theta \ll x, \rho(\mathfrak{r} x)<\frac{1}{2}$, and

$$
\mathfrak{q}(S(u), T(v)) \ll x[\mathfrak{q}(u, S(v))+\mathfrak{q}(v, T(u))], \quad \forall u \in A, v \in B .
$$

Then $\mathrm{S}$ and $\mathrm{T}$ have a single common fixed point.

Proof. As in Theorem 2.2, let $\mathfrak{u}_{0}$ be arbitrarily chosen element in $\mathfrak{C}$, if $\mathfrak{u}_{0} \in B$, then $\mathfrak{u}_{1}=T\left(u_{0}\right) \in A$, $u_{2}=S\left(u_{1}\right) \in B, u_{3}=T\left(u_{2}\right) \in A$, and then define $\left\{u_{n}\right\}_{n \in N}$ by induction:

$$
u_{2 n+2}=S\left(u_{2 n+1}\right) \in B \text { and } u_{2 n+1}=T\left(u_{2 n}\right) \in A, \quad \forall n \geqslant 0 .
$$

First, suppose $n$ is an odd natural number. Then

$$
\begin{aligned}
\mathfrak{q}\left(u_{n+1}, u_{n}\right) & =\mathfrak{q}\left(S\left(u_{n}\right), T\left(u_{n-1}\right)\right) \\
& \ll x\left[\mathfrak{q}\left(u_{n}, S\left(u_{n-1}\right)\right)+\mathfrak{q}\left(u_{n-1}, T\left(u_{n}\right)\right)\right] \\
& \ll x\left[\mathfrak{q}\left(u_{n}, u_{n}\right)+\mathfrak{q}\left(u_{n-1}, u_{n+1}\right)\right] \\
& \ll x \mathfrak{q}\left(u_{n-1}, u_{n+1}\right) \\
& \ll x \mathfrak{r}\left[\mathfrak{q}\left(u_{n-1}, u_{n}\right)+\mathfrak{q}\left(u_{n}, u_{n+1}\right)\right] \\
& \ll x \mathfrak{r} \mathfrak{q}\left(u_{n-1}, u_{n}\right)+x \mathfrak{r} \mathfrak{q}\left(u_{n}, u_{n+1}\right) .
\end{aligned}
$$

Consequently

$$
\mathfrak{q}\left(u_{n+1}, u_{n}\right)-x \mathfrak{r} \mathfrak{q}\left(u_{n}, u_{n+1}\right) \ll x \mathfrak{r} \mathfrak{q}\left(u_{n-1}, u_{n}\right), \quad[\Delta-x \mathfrak{r}] \mathfrak{q}\left(u_{n}, u_{n+1}\right) \ll x \mathfrak{r q}\left(u_{n-1}, u_{n}\right) .
$$

Hence

$$
\mathfrak{q}\left(u_{n}, u_{n+1}\right) \ll[\Delta-x \mathfrak{r}]^{-1} x \mathfrak{r} \mathfrak{q}\left(u_{n-1}, u_{n}\right) .
$$

Let $\mathfrak{z}=[\Delta-\chi \mathfrak{r}]^{-1} \chi \mathfrak{r}$ and continue in this process, we see that

$$
\mathfrak{q}\left(u_{n}, u_{n+1}\right) \ll \mathfrak{z}^{n} \mathfrak{q}\left(u_{1}, u_{n_{0}}\right) .
$$

Similar conclusion can be proved for any even natural number $\mathfrak{n}$. Using Lemma 2.4 with $\mathfrak{y}_{\mathfrak{o}}=x \mathfrak{r}, \rho(\mathfrak{z})<\frac{1}{2}$ insures that that the sequence $\left\{\mathfrak{u}_{n}\right\}_{\mathfrak{n} \in N}$ is Cauchy sequence. By the completeness of $\mathfrak{C}$, there is $w \in \mathfrak{C}$ such that $\lim _{n \rightarrow \infty} u_{n}=w$. We have

$$
\lim _{n \rightarrow \infty} S\left(u_{2 n+1}\right)=w \text { and } \lim _{n \rightarrow \infty} T\left(u_{2 n}\right)=w .
$$

Now

$$
\begin{aligned}
\mathfrak{q}\left(u_{2 n+2}, T(w)\right) & =\mathfrak{q}\left(S\left(u_{2 n+1}\right), T(w)\right) \\
& \ll x\left[\mathfrak{q}\left(u_{2 n+1}, S(w)\right)+\mathfrak{q}\left(w, T\left(u_{2 n+1}\right)\right)\right] \\
& \ll x\left[\mathfrak{q}\left(T\left(u_{2 n}\right), S(w)\right)+\mathfrak{q}\left(u_{2 n+2}, w\right)\right] \\
& \ll x\left[x\left\{\mathfrak{q}\left(u_{2 n}, T(w)\right)+\mathfrak{q}\left(S\left(u_{2 n}\right), w\right)\right\}+\mathfrak{q}\left(u_{2 n+2}, w\right)\right] \\
& \ll x\left[x\left\{\mathfrak{q}\left(u_{2 n}, T(w)\right)+\mathfrak{q}\left(u_{2 n+1}, w\right)\right\}+\mathfrak{q}\left(u_{2 n+2}, w\right)\right] .
\end{aligned}
$$


On limiting approach as $n \rightarrow \infty$, equation (2.7) proves that:

$$
\mathfrak{q}(w, T(w)) \ll x^{2} \mathfrak{q}(T(w), w) .
$$

Hence $\left(\Delta-x^{2}\right) \mathfrak{q}(w, T(w)) \ll \Theta$, multiplying the two sides with $\left(\Delta-x^{2}\right)^{-1}$, we get $\mathfrak{q}(w, T(w))=\Theta$, that is $\mathrm{T}(w)=w$ and $w$ is a fixed point of T. Similarly, we prove that $S(w)=w$. If there exists another point $y$ such that $S(y)=y=T(y)$, then we get

$$
\begin{aligned}
\mathfrak{q}(w, y)=\mathfrak{q}(S(w), T(y)) & \ll x[\mathfrak{q}(w, S(y))+\mathfrak{q}(y, T(w))] \\
& \ll x[\mathfrak{q}(w, y)+\mathfrak{q}(y, w)] \ll 2 x \mathfrak{q}(w, y) \\
& \ll 2 x \mathfrak{r}[\mathfrak{q}(w, w)+\mathfrak{q}(w, y)] \ll 2 x \mathfrak{r} \mathfrak{q}(w, y) .
\end{aligned}
$$

Thus

$$
\mathfrak{q}(w, y)-2 x \mathfrak{r} \mathfrak{q}(w, y) \ll \Theta ;[\Delta-2 x \mathfrak{r}] \mathfrak{q}(w, y) \ll \Theta .
$$

Since $\rho(x \mathfrak{r})<\frac{1}{2}$, we have $\rho(2 x \mathfrak{r})<1$ and $\Delta-2 \chi \mathfrak{r}$ has an inverse, multiplying the two sides with $(\Delta-2 x \mathfrak{r})^{-1}$ gives $\mathfrak{q}(w, y) \ll \Theta$, that is $\mathfrak{q}(w, y)=\Theta$, consequently $w=y$.

Corollary 2.7. If $\mathrm{S}$ is a cyclic Banach algebra Kannan type mapping on a complete cone quasi metric space $\mathfrak{C}$ with respect to $(A, B)$, then $S$ has only one fixed point.

\section{Conclusion}

This paper proves the existence of a single common fixed point of two self mappings defined on complete cone quasi metric space with respect to Banach algebra and then it proves the existence of single fixed point of generalized cyclic Banach algebra contraction and cyclic Banach algebra Kannan type on cone quasi metric space $\mathfrak{C}$ with respect to a pair of non empty subsets $(A, B)$ of $\mathfrak{C}$. Consequently; generalizations of some fixed point theorems proved in the usual classical metric spaces.

\section{Acknowledgment}

The author is sincerely thank the anonymous referees and editors for their valuable comments which gave a step forward to the main manuscript file.

\section{References}

[1] M. Abbas, V. Ćojbašić Rajić, T. Nazir, S. Radenović, Common fixed point of mappings satisfying rational inequalities in ordered complex valued generalized metric spaces, Afr. Mat., 26 (2015), 17-30. 1

[2] S. M. Ali, Fixed point theorems of $\{a, b, c\}$ contraction and nonexpansive type mappings in weakly Cauchy normed spaces, Anal. Theory Appl., 29 (2013), 280-288. 1, 1.5

[3] S. M. Ali Abou Bakr, Some Generalized Fixed Point Theorems of Contraction Type Mappings in Quasi Metric Spaces, J. Math. Stat., 14 (2017), 319-324. 1, 1.5

[4] H. Aydi, E. Karapinar, B. Samet, Remarks on some recent fixed point theorems, Fixed Point Theory Appl., 2012 (2012), 6 pages. 1

[5] H. Çakally, A. Sönmez, Ç. Genç, On an equivalence of topological vector space valued cone metric spaces and metric spaces, Appl. Math. Lett., 25 (2012), 429-433. 1

[6] W.-S. Du, A note on cone metric fixed point theory and its equivalence, Nonlinear Anal., 72 (2010), 2259-2261. 1

[7] P. N. Dutta, B. S. Choudhury, A generalisation of contraction principle in metric spaces, Fixed Point Theory Appl., 2008 (2008), 8 pages. 1

[8] E. El-Shobaky, S. M. Ali, M. S. Ali, Generalization of Banach contraction principle in two directions, J. Math. Stat., 3 (2007), 112-115. 1.5

[9] J. Fernandez, N. Malviya, S. Radenovič, K. Saxena, F-cone metric spaces over Banach algebra, Fixed Point Theory Appl., 2017 (2017), 18 pages. 1

[10] J. Fernandez, G. Modi, N. Malviya, Some fixed point theorems for contractive maps in N-cone metric spaces, Math. Sci. (Springer), 9 (2015), 33-38. 1 
[11] J. Fernandez, K. Saxena, N. Malviya, Fixed points of expansive maps in partial cone metric spaces, Gazi University J. Sci., 27 (2014), 1085-1091. 1

[12] R. George, H. A. Nabwey, R. Rajagopalan, S. Radenović, K. P. Reshma, Rectangular cone b-metric spaces over Banach algebra and contraction principle, Fixed Point Theory Appl., 2017 (2017), 15 pages. 1

[13] R. H. Haghi, S. Rezapour, N. Shahzad, Some fixed point generalizations are not real generalizations, Nonlinear Anal., 74 (2011), 1799-1803. 1

[14] R. H. Haghi, S. Rezapour, N. Shahzad, Be careful on partial metric fixed point results, Topology Appl., 160 (2013), 450-454. 1

[15] H. P. Huang, S. Radenović, Some fixed point results of generalised Lipschitz mappings on cone b-metric spaces over Banach algebras, J. Comput. Anal. Appl., 20 (2016), 566-583. 1

[16] L.-G. Huang, X. Zhang, Cone metric spaces and fixed point theorem of contractive mappings, J. Math. Anal. Appl., 332 (2007), 1468-1476. 1

[17] W. A. Kirk, P. S. Srinivasan, P. Veeramani, Fixed points for mappings satisfying cyclical contractive conditions, Fixed Point Theory, 4 (2003), 79-89. 1, 1

[18] H. Liu, S. Y. Xu, Cone metric spaces with Banach algebras and fixed point theorems of generalized Lipschitz mappings, Fixed Point Theory Appl., 2013 (2013), 10 pages. 1, 1, 1.1

[19] N. Malviya, B. Fisher, N-cone metric space and fixed points of asymptotically regular maps, Filomat, 2013 (2013), 11 pages. 1

[20] W. Rudin, Functional Analysis, McGraw-Hill, New York, (1991). 1

[21] N. Sharma, Fixed point theorem in cone B-metric spaces using contractive mappings, Global J. Pure Appl. Math., 13 (2017), 2997-3004. 1 